\title{
Long non-coding RNA PCGEM1 as a biomarker for prostate cancer
}

\author{
Wei Yang ${ }^{\mathrm{a}}$, Fawei $\mathrm{He}^{\mathrm{a}}$, Yuan $\mathrm{Li}^{\mathrm{a}}$, Yangkui Zhai ${ }^{\mathrm{b}}$, Bo Tan ${ }^{\mathrm{a}}$, Haixiang $\mathrm{Wu}^{\mathrm{a}, *}$ \\ a Department of Ultrasonics, Sichuan Provincial Cancer Hospital, 610041, Sichuan, China \\ b Metabolic Disease Hospital, Tianjin Medical University, 300070, Tianjian, China
}

*Corresponding author, e-mail: HXW_1971@163.com

Received 21 Apr 2015

Accepted 3 Jun 2016

\begin{abstract}
Prostate cancer (PC) is the second most common diagnosed malignant disease. Long non-coding RNAs (lncRNAs), which account for approximately $98 \%$ of the human genome, are becoming increasingly interesting with regard to various diseases and have great potential for diagnosis and prognostic monitoring of PC. To identify a new diagnosis marker for metastatic PC, we enrolled a total of 144 patients with PC, including 57 metastatic and 87 localized PC patients, and 148 healthy subjects. Patients were followed up routinely at 3-month intervals for 5 years. The expression of 10 selected lncRNAs in peripheral blood from participants was measured. Among the 10 selected lncRNAs, the expression of PCGEM1 in the metastatic group was 2.57 times that in the localized group $(p<0.001)$ and 2.96 times that in the control group $(p<0.001)$. Patients with higher AJCC stage had significantly elevated PCGEM1 expression (one-way ANOVA, $p<0.001$ ). The relative expression of PCGEM1 in patients with higher Gleason score was also higher than that in patients with lower Gleason score $(p=0.003)$. Moreover, patients with more than 5 years survival time had significantly lower PCGEM1 expression than the rest $(p=0.017)$ and patients with elevated PCGEM1 relative expression had significantly shorter survival time $(p<0.001)$. The present study suggested that PCGEM1 expression in peripheral blood could act as a diagnostic marker of metastatic PC. It would also be a prognostic marker to predict the survival time.
\end{abstract}

KEYWORDS: peripheral blood, survival time, diagnostic, prognostic

\section{INTRODUCTION}

Prostate cancer (PC) is the second most common diagnosed malignant disease and the sixth leading cause for cancer related death among men worldwide. It was estimated that there were 899000 new cases and 258000 deaths in 2008 worldwide ${ }^{1}$. With the use of prostate-specific antigen in the serum, the proper rate of PC diagnosis increased over the past decades ${ }^{2}$; however, this procedure is limited by false-negative biopsies and overdiagnosis of clinically insignificant malignancies ${ }^{3}$.

The ENCODE project has recently reported that more than $90 \%$ of the human genome can be transcribed and approximately $98 \%$ of these transcripts were of no protein-coding capacity, among which long non-coding RNAs (lncRNAs) are the most common type ${ }^{4}$. They are arbitrarily defined as being more than $200 \mathrm{nt}$ in length and are involved in regulating a wide variety of important cellular functions, such as genome imprinting, gene expression, recruitment of chromatin modifying machinery, and regulation of $\mathrm{X}$ chromosome inactivation ${ }^{5}$.
Notably, lncRNAs were found to be specifically regulated, suggesting the potential to serve as novel biomarkers and therapeutic targets ${ }^{6}$. Until now, many lncRNAs were detected in PC patients with specific expression pattern and were suggested to be biomarker of PC. For example, lncRNA PCA3 was found to be strongly expressed in more than $95 \%$ of primary PC specimens and was not expressed in other normal human tissues ${ }^{7}$. The PROGENSA PCA3 test is the first urine-based molecular diagnostic test for PC which was approved by American $\mathrm{FDA}^{8}$. The lncRNA metastasis-associated lung adenocarcinoma transcript 1 (MALAT1) was another marker of $\mathrm{PC}$, the expression of which was correlated with poor prognosis in PC patients ${ }^{9}$. Crea et al found a total of 153 upregulated and 77 downregulated lncRNAs in metastatic versus nonmetastatic xenografts and focused on PCAT18 for biomarker analysis ${ }^{10}$. Based on the expression profiles, we investigated the expression pattern of the 10 most dysregulated lncRNAs in our blood samples of PC patients and normal controls, and found PCGEM1 could serve as a new diagnosis marker for metastatic 
prostate cancer for AJCC for the following reasons: (1) the expression of PCGEM1 was higher in metastatic group than that in localized and control groups ( $p<0.001)$; (2) PC patients with higher AJCC stage had significantly elevated PCGEM1 expression $(p<0.001)$; (3) PC patients with lower PCGEM1 showed prolonged survival time.

\section{MATERIALS AND METHODS}

\section{Study population}

A total of 292 participants, which included 144 consecutive patients who had a histopathologic diagnosis of PC and 148 geographically and sexually matched healthy controls, were recruited from outpatient Department of the Second Hospital of Sichuan. Detailed clinical and pathology data were obtained from physician records and hospital notes. Among the 144 patients, cancer metastasis was diagnosed in 57 patients (40\%); the metastatic sites included lymph nodes, bones, and other distant organs. All patients were followed-up at 3-month intervals for 5 years, routinely. At each visit, a comprehensive examination was carried out. Survival time was measured from the date of histopathology diagnosis until the date of death or last follow-up. Two or more pathologists classified the stages of PC patients according to the American Joint Committee on cancer (AJCC), 7th edition ${ }^{11}$. Each subject signed written informed consent to participate in the study. This study was approved by the Institutional Review Board of the Second Hospital of Sichuan (project no. B-133).

\section{RNA samples}

At the first visit, $5 \mathrm{ml}$ venous blood samples of each subject were collected into a blood collection tube. Total RNA was extracted from lymphocytes using PAXgene RNA collection tubes (QIAGEN, USA). The concentration of RNA samples was quantified using a NanoDrop ND-1000 spectrophotometer; the quality of RNA was assessed with denaturing agarose gel electrophoresis.

\section{Real-time PCR}

First, total RNA was reversely transcribed to cDNA with PrimeScript RT reagent Kit equipped with gDNA Eraser (TaKaRa, China) strictly according to the manufacturer's instructions. Then, real-time PCR was performed using SsoFast EvaGreen Supermix (Bio-Rad, USA) on a CFX96 Real-Time PCR Detection System (Bio-Rad, USA).
The PCR procedures included an initial step at $95^{\circ} \mathrm{C}$ for $30 \mathrm{~s}$, followed by 40 cycles of amplification and quantification $\left(95^{\circ} \mathrm{C}\right.$ for $5 \mathrm{~s}, 60^{\circ} \mathrm{C}$ for $\left.5 \mathrm{~s}\right)$. Each cDNA sample was performed in triplicate in a final volume of $25 \mu \mathrm{l}$ containing $1 \mu \mathrm{l}$ of cDNA and $400 \mathrm{nmol}$ of forward and reverse gene-specific primers.

Relative gene expression level was quantified based on the cycle threshold $(\mathrm{Ct})$ values, where $G A P D H$ was used as an internal control whose expression was stable in primary and metastatic $\mathrm{PC}^{10}$. For quantitative results, expression of each gene was represented as a fold change using the following mathematical model:

$$
\text { fold change }=\frac{\left(E_{\text {target }}\right)^{\Delta \mathrm{Ct} \mathrm{t}_{\text {target }}}}{\left(E_{\text {ref }}\right)^{\Delta \mathrm{Ct}_{\text {ref }}}}
$$

where $E_{\text {target }}$ and $E_{\text {ref }}$ are the PCR efficiency of target gene transcript and reference gene transcript, respectively; $\Delta \mathrm{Ct}_{\text {target }}$ and $\Delta \mathrm{Ct}_{\text {ref }}$ are the $\mathrm{Ct}$ deviation of control minus sample of the target gene transcript and the reference gene transcript, respectively ${ }^{12}$. All primer pairs are available upon request.

\section{Statistical analysis}

The programs GRAPHPAD PRISM 5, SPSS 17.0, and Microsoft OfFICE EXCEL 2007, were used for data analysis. The data are presented as mean \pm SD for continuous variables or percentages for categorical variables. Specific analysis methods were used with detailed description. A value of $p<0.05$ indicated a statistically significant result.

\section{RESULTS}

\section{Cohort characteristics}

A total of 292 subjects, including 144 BC patients (aged between 38 and 81, median 72 years) and 148 age- and gender-matched healthy controls (aged between 42 and 77, median 67 years) were enrolled in the present study. The clinical characteristics of all participants are summarized in Table 1 . The distribution of race and marital status were compatible in both groups.

\section{Real-time PCR validation of 10 lncRNAs candidates}

Through RNA sequencing, Crea et al identified 153 upregulated and 77 downregulated lncRNAs in metastatic xenografts, compared to nonmetastatic xenografts $^{10}$. We selected 10 lncRNAs with most drastic expression fluctuation for validation 
Table 1 Characteristics of the study participants.

\begin{tabular}{lccc}
\hline Characteristics & $\begin{array}{c}\text { Bladder } \\
\text { cancer } \\
(n=144)\end{array}$ & $\begin{array}{c}\text { Healthy } \\
\text { population } \\
(n=148)\end{array}$ & $p$ value \\
\hline $\begin{array}{l}\text { Age (median, range) } \\
\text { (years) }\end{array}$ & $72(38-81)$ & $67(42-77)$ & 0.271 \\
Race: & $132(92 \%)$ & $139(94 \%)$ & 0.456 \\
$\quad$ Han ( $n, \%)$ & $12(8 \%)$ & $9(6 \%)$ & \\
$\quad$ Non-Han (n, \%) & $119(83 \%)$ & $124(77 \%)$ & 0.794 \\
Marital status: & $25(17 \%)$ & $24(23 \%)$ & \\
$\quad$ Married $(n, \%)$ & $96(67 \%)$ & $43(34 \%)$ & $<0.001$ \\
$\quad$ Not married $(n, \%)$ & & & \\
Smoker $(n, \%)$ & & & \\
\hline
\end{tabular}

in our cohorts consisting of 20 metastatic samples and 20 nonmetastatic samples. The 10 lncRNAs included 7 upregulated lncRNAs, namely GTF2IRD2P1, C3orf51, LOC339535, LOC285692, PCGEM1, SH3GL1P1, and TYRO3P; and 3 downregulated lncRNAs, namely LOC100132215, FLJ37307, and $A M Z 2 P 1$. A general consistent result of each lncRNA between our cohort and the cohort used by Crea was confirmed in 7 lncRNAs in terms of regulation direction (upregulation or downregulation) and significance except LOC285692, LOC100132215 and AMZ2P1 (Fig. 1). Among the 7 lncRNAs, PCGEM1 expression in metastatic group was 2.57 times compared to the expression in nonmetastatic group. We therefore focused on this lncRNA in further analysis.

\section{PCGEM1 expression was positively correlated with PC progression}

We further investigated the expression of PCGEM1 in another large cohort consisting of 148 healthy controls, 59 metastatic PC patients and 85 localized
Table 2 Relative expression of PCGEM1 in PC patients of different AJCC stages and Gleason score.

\begin{tabular}{lccc}
\hline & $\begin{array}{c}\text { Patients } \\
(n, \%)\end{array}$ & $\begin{array}{c}\text { Relative expression } \\
\text { of PCGEM1 }\end{array}$ & $p$ value \\
\hline AJCC stage: & & & \\
$\quad$ I/II & $31(22 \%)$ & $1.00 \pm 0.37$ & \\
III & $29(20 \%)$ & $1.49 \pm 0.88$ & $<0.001$ \\
$\quad$ IV & $84(58 \%)$ & $3.9 \pm 1.3$ & \\
Gleason score: & & & \\
$\quad \leqslant 5$ & $9(6 \%)$ & $1.00 \pm 0.29$ & \\
6 & $16(11 \%)$ & $1.7 \pm 1.0$ & 0.003 \\
7 & $58(40 \%)$ & $1.92 \pm 0.92$ & \\
$\geqslant 8$ & $61(42 \%)$ & $4.2 \pm 1.9$ & \\
Tumour metastasis: & & & \\
$\quad$ Metastasis & $57(40 \%)$ & $1.00 \pm 0.49$ & $<0.001$ \\
$\quad$ Localized & $87(60 \%)$ & $3.5 \pm 1.7$ & \\
\hline
\end{tabular}

PC patients (Table 2). The results showed that the expression of PCGEM1 was higher in metastatic group than that in localized and control groups ( $p<$ 0.001, Fig. 2).

According to AJCC, we divided all the PC patients into 4 stages, namely I, II, III, and IV stage. The number of patients and the relative expression of PCGEM1 are described in Table 2. A total of 31 patients (22\%) were grouped into stage I and II, $29(20 \%)$ in group III, and $84(58 \%)$ in group IV. Patients with higher AJCC stages had significantly elevated PCGEM1 expression (one-way ANOVA, $p<$ 0.001 ). The relative expression in patients with different Gleason scores $(\leqslant 5,6,7$, and $\geqslant 8$ ) were also investigated, of which the result was consistent to the result reflected by AJCC stage (one-way ANOVA, $p=0.003$ ).

Besides, the cases with tumour metastasis had significantly higher PCGEM1 expression compared with those with localized tumours (non-paired

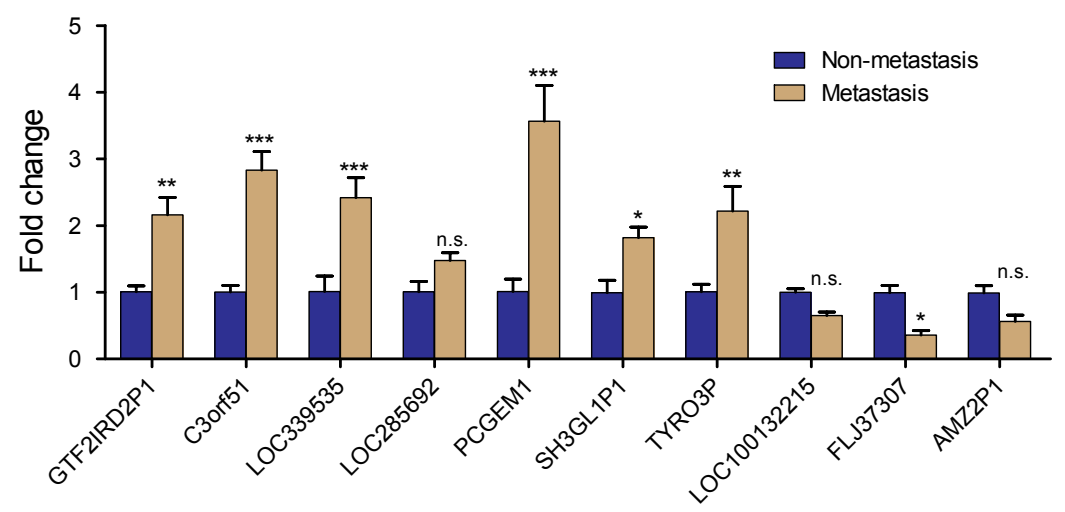

Fig. 1 Real-time PCR validation of $10 \mathrm{lncRNAs}$ candidates. Fold changes were calculated by the $2^{-\Delta \Delta \mathrm{Ct}}$ method. The data were analysed using two-way ANOVA. The bars represent the means \pm SEM $(n=20) . * p<0.05$, ** $p<0.01$, and $* * * p<0.001$, which were determined using Bonferroni's post-hoc test, represented significant differences; n.s., not significant. 


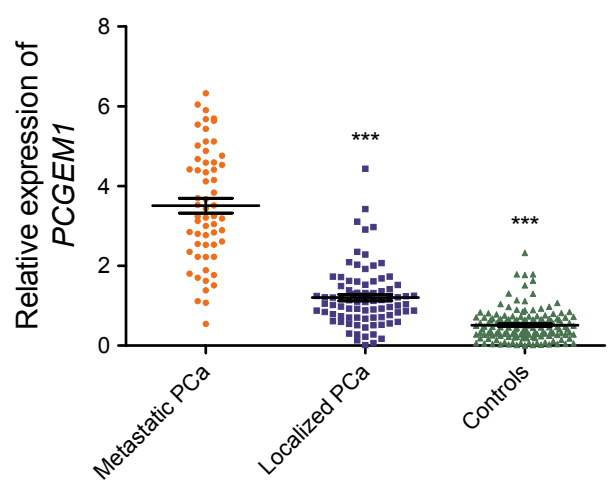

Fig. 2 Relative expression of PCGEM1 in peripheral blood in metastatic PC, localized PC, and control groups. Fold changes were calculated by the $2^{-\Delta \Delta \mathrm{Ct}}$ method. Error bars represent means \pm SEM. The data were analysed using one-way ANOVA. $* * * p<0.001$, which were determined using Turkey multiple comparison test, represent significant differences.

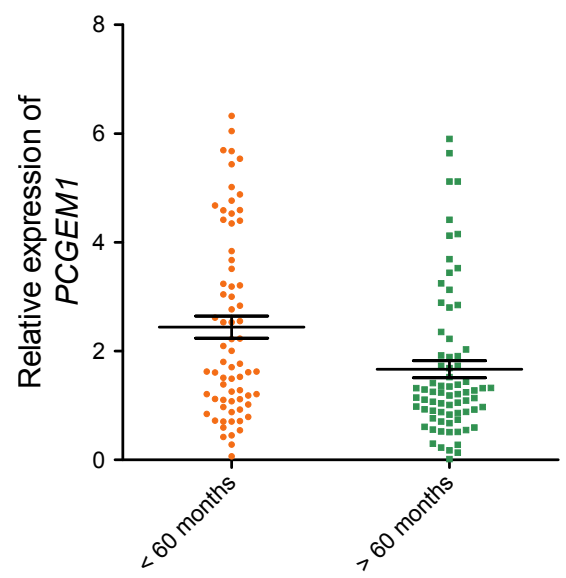

Fig. 3 PC patients surviving over 5 years had lower PCGEM1 relative expression. Comparison between two groups was performed by Mann-Whitney test. Survival time: the time from the date of histopathology diagnosis to the date of death.

t-test, $p<0.001)$.

\section{Patients with lower PCGEM1 expression showed prolonged survival time}

To analyse the association of PCGEM1 expression with patients' survival time, we first compared the relative PCGEM1 expression in those with survival time more than 5 years and those died in recent 5 years. The result showed that patients with more than 5 years survival time had significantly lower PCGEM1 expression than the rest $(p=0.017$, Fig. 3).

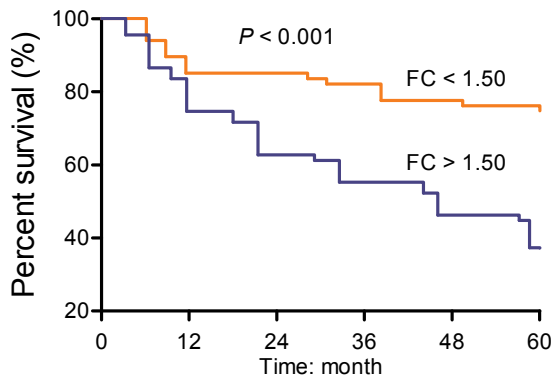

Fig. 4 PC patients with lower PCGEM1 relative expression had longer survival time.

We further divided all the cases into two groups according to the relative PCGEM1 expression to controls, one with elevated PCGEM1 relative expression $(>1.5, n=76)$ and the other with lower PCGEM1 relative expression $(<1.5, n=68)$. Survival curve analysis revealed that patients with elevated PCGEM1 relative expression had significantly shorter survival time $(p<0.001)$ and were at 2.54 times higher risk of death compared with those with lower PCGEM1 relative expression $(p<0.001$, Fig. 4).

\section{DISCUSSION}

In recent years, the functions of lncRNAs in different diseases have been widely investigated and reported. For example, spinocerebellar ataxia type 8 (SCA8) patients have a trinucleotide expansion in ataxin 8 opposite strand (ATXN8OS), a lncRNA that is antisense to the KLHL1 gene ${ }^{13}$. High expression levels of lncRNA HOTAIR has been shown to be an independent prognostic factor of hepatocellular carcinoma after liver transplantation ${ }^{14}$. In PC students, dysregulation of lncRNAs was noticed in several studies. However, we found that most of these studies focused on tumour tissues, but not peripheral blood, for lncRNAs analysis, which conferred big trouble for diagnosis and prognostic monitoring of PC. If dysregulated expression of certain lncRNAs can be detected in peripheral blood, it may bring great convenience for PC diagnosis.

In the present study, we found that the expression of PCGEM1 in the metastatic group was significantly higher than that in the localized and control groups. Besides, patients with higher AJCC stages had significantly elevated PCGEM1 expression, compared to those with lower AJCC stages. Moreover, patients with more than 5 years survival time had significantly lower PCGEM1 expression than the rest; and vice versa, patients with elevated PCGEM1 
relative expression had significantly shorter survival time. All the abovementioned evidence suggested that PCGEM1 could act as a potential biomarker for PC diagnosis and prognostic monitoring. Besides, PCGEM1 could potentially become a part of prognosticating tool along with GPS and Polaris biopsy PCR kits to determine suitability of men on active surveillance.

In previous studies, PCGEM1 was identified as a prostate cancer specific lncRNA that is capable of promoting proliferation and inhibiting apoptosis. PCGEM1 overexpression in LNCaP cells results in delayed induction of p53 and p21 induced by doxorubicin, which could therefore inhibit cell apoptosis ${ }^{15}$. Besides, PCGEM1 is an in vivo androgenregulated transcript with potential nuclear and/or cytoplasmic function ${ }^{16}$. Although the involvement of PCGEM1 in PC was widely reported, the role of PCGEM1 in PC is still controversial. For example, androgen deprivation is able to induce PCGEM1 and causes its accumulation in nuclear speckles ${ }^{17}$, while this was not the case in another study which revealed that PCGEM1 was stimulated by androgen and downregulated by castration in xenograft models ${ }^{16}$. Similarly, PCGEM1 along with PRNCR1 can impact AR signalling through interaction with AR to promote castration resistance ${ }^{18}$, however, this is not supported by a comprehensive analysis of RNA-sequencing data ${ }^{19}$. Genetic analysis also supported the involvement of PCGEM1 in PC risk. PCGEM1 polymorphisms might contribute to $P C$ risk in Chinese men and SNP rs6434568 C carriers or rs 16834898 C carriers had significantly decreased risk of $\mathrm{PC}^{20}$.

For real-time PCR validation, we only selected 10 lncRNA candidates. In fact, many other lncRNAs surpassing the lncRNA list in our study could be potential biomarker for PC, since as many as 153 upregulated and 77 downregulated lncRNAs have been identified ${ }^{10}$. It is quite meaningful to investigate the potential of other lncRNAs to be biomarker of PC.

Another issue to be considered is the accuracy of a single molecule to diagnose diseases. In fact, many studies showed that combinational usage of several molecules could be more efficient for diagnosis and prognostic monitoring of diseases. Meng et $\mathrm{al}^{21}$ showed that a four-lncRNA (U79277, AK024118, BC040204, AK000974) expression signature could be used to predict breast cancer survival $^{21}$. Using a risk score based on the expression signature of these lncRNAs, the authors separated the patients into low-risk and high-risk groups with significantly different survival times, which was validated in three other cohorts ${ }^{21}$. Li et $\mathrm{al}^{22}$ identified a three-lncRNA signature (including the lncRNAs ENST00000435885.1, XLOC_013014, and ENST00000547963.1) that was able to classify the oesophageal squamous cell carcinoma patients into two groups with significantly different overall survival $^{22}$. In a future study, we could investigate using lncRNA combination(s) to diagnose PC.

\section{REFERENCES}

1. Center MM, Jemal A, Lortet-Tieulent J, Ward E, Ferlay J, Brawley O, Bray F (2012) International variation in prostate cancer incidence and mortality rates. Eur Urol 61, 1079-92.

2. Catalona WJ, Smith DS, Ratliff TL, Dodds KM, Coplen DE, Yuan JJJ, Petros JA, Andriole GL (1991) Measurement of prostate-specific antigen in serum as a screening-test for prostate-cancer. New Engl J Med 324, 1156-61.

3. Schröder FH, Hugosson J, Roobol MJ, Tammela TL, Ciatto S, Nelen V, Kwiatkowski M, Lujan M, et al (2009) Screening and prostate-cancer mortality in a randomized European study. New Engl J Med 360, 1320-8.

4. Birney E, Stamatoyannopoulos JA, Dutta A, Guigo R, Gingeras TR, Margulies EH, Weng Z, Snyder M, et al (2007) Identification and analysis of functional elements in $1 \%$ of the human genome by the ENCODE pilot project. Nature 447, 799-816.

5. Hung T, Chang HY (2010) Long noncoding RNA in genome regulation: prospects and mechanisms. RNA Biol 7, 582-5.

6. Gibb EA, Brown CJ, Lam WL (2011) The functional role of long non-coding RNA in human carcinomas. Mol Canc 10, 38.

7. Hessels D, Klein Gunnewiek JMT, van Oort I, Karthaus HFM, van Leenders GJL, van Balken B, Kiemeney LA, Witjes JA, et al (2003) DD3 ${ }^{\mathrm{PCA}^{3}}$-based molecular urine analysis for the diagnosis of prostate cancer. Eur Urol 44, 8-15.

8. Hessels D, Schalken JA (2009) The use of PCA3 in the diagnosis of prostate cancer. Nat Rev Urol 6, 255-61.

9. Ren SC, Liu YW, Xu WD, Sun Y, Lu J, Wang F, Wei M, Shen J, et al (2013) Long noncoding RNA MALAT-1 is a new potential therapeutic target for castration resistant prostate cancer. J Urol 190, 2278-87.

10. Crea F, Watahiki A, Quagliata L, Xue H, Pikor L, Parolia A, Wang Y, Lin D, et al (2014) Identification of a long non-coding RNA as a novel biomarker and potential therapeutic target for metastatic prostate cancer. Oncotarget 5, 764-74.

11. Edge SB, Compton CC (2010) The American Joint Committee on Cancer: the 7th edition of the AJCC Cancer Staging Manual and the future of TNM. Ann Surg Oncol 17, 1471-4. 
12. Pfaffl MW (2001) A new mathematical model for relative quantification in real-time RT-PCR. Nucleic Acids Res 29, e45.

13. Moseley ML, Zu T, Ikeda Y, Gao W, Mosemiller AK, Daughters RS, Chen G, Weatherspoon MR, et al (2006) Bidirectional expression of CUG and CAG expansion transcripts and intranuclear polyglutamine inclusions in spinocerebellar ataxia type 8. Nat Genet 38, 758-69.

14. Yang Z, Zhou L, Wu L, Lai M, Xie H, Zhang F, Zheng $S$ (2011) Overexpression of long non-coding RNA HOTAIR predicts tumor recurrence in hepatocellular carcinoma patients following liver transplantation. Ann Surg Oncol 18, 1243-50.

15. Fu X, Ravindranath L, Tran N, Petrovics G, Srivastava S (2006) Regulation of apoptosis by a prostatespecific and prostate cancer-associated noncoding gene, PCGEM1. DNA Cell Biol 25, 135-41.

16. Parolia A, Crea F, Xue H, Wang Y, Mo F, Ramnarine VR, Liu HH, Lin D, et al (2015) The long non-coding RNA PCGEM1 is regulated by androgen receptor activity in vivo. Mol Canc 14, 46.

17. Zhang Z, Zhou N, Huang J, Ho TT, Zhu Z, Qiu Z, Zhou X, Bai C, et al (2016) Regulation of androgen receptor splice variant AR3 by PCGEM1. Oncotarget 7, 15481-91.

18. Yang L, Lin C, Jin C, Yang J, Tanasa B, Li W, Merkurjev D, Ohgi KA, et al (2013) lncRNA-dependent mechanisms of androgen-receptor-regulated gene activation programs. Nature 500, 598-602.

19. Prensner JR, Sahu A, Iyer MK, Malik R, Chandler B, Asangani IA, Poliakov A, Vergara IA, et al (2014) The lncRNAs PCGEM1 and PRNCR1 are not implicated in castration resistant prostate cancer. Oncotarget $\mathbf{5}$, 1434-8.

20. Xue Y, Wang M, Kang M, Wang Q, Wu B, Chu H, Zhong D, Qin C, et al (2013) Association between lncRNA PCGEM1 polymorphisms and prostate cancer risk. Prostate Canc Prostatic Dis 16, 139-44.

21. Meng J, Li P, Zhang Q, Yang Z, Fu S (2014) A fourlong non-coding RNA signature in predicting breast cancer survival. $J$ Exp Clin Canc Res 33, 84.

22. Li J, Chen Z, Tian L, Zhou C, He M, Gao Y, Wang S, Zhou F, et al (2014) LncRNA profile study reveals a three-lncRNA signature associated with the survival of patients with oesophageal squamous cell carcinoma. Gut 63, 1700-10. 